\title{
THE DEGREES OF CATEGORICAL THEORIES WITH RECURSIVE MODELS
}

\author{
URI ANDREWS
}

(Communicated by Julia Knight)

\begin{abstract}
We show that even for categorical theories, recursiveness of the models guarantees no information regarding the complexity of the theory. In particular, we show that every tt-degree reducible to $0^{(\omega)}$ contains both $\aleph_{1^{-}}$ categorical and $\aleph_{0}$-categorical theories in finite languages, all of whose countable models have recursive presentations.
\end{abstract}

\section{INTRODUCTION}

A fundamental question of recursive model theory is to understand the relationship between the complexity of a theory and the complexity of presentations of its models. It is well known that if a theory is recursive, then the Henkin construction produces a decidable model, that is, a model whose elementary diagram is recursive. Also, if $T$ is recursive and $\aleph_{1}$-categorical, then all of its countable models are decidably presentable [7, [10. On the other hand, if $T$ has a recursive model, that is, a model whose atomic diagram is recursive, then in general we can only say that $T$ is tt-reducible to $0^{(\omega)}$. Naturally, one would like to know whether this bound can be improved upon for tame theories. Two natural classes of tame theories are the $\aleph_{0}$-categorical and $\aleph_{1}$-categorical theories.

Goncharov and Khoussainov [5] showed that for each $n$ there is an $\aleph_{1}$-categorical, non- $\aleph_{0}$-categorical theory Turing equivalent to $0^{(n)}$ all of whose countable models have recursive presentations. They also showed that for each $n$ there is an $\aleph_{0}$-categorical theory Turing equivalent to $0^{(n)}$ with a recursive countable model. Fokina [4] extended these results from $0^{(n)}$ to any arithmetical Turing degree. Goncharov and Khoussainov conclude by asking whether there is a theory Turing equivalent to $0^{(\omega)}$ which is $\aleph_{1}$-categorical and all of its countable models are recursive, and also whether there is an $\aleph_{0}$-categorical theory Turing equivalent to $0^{(\omega)}$ with a recursive countable model.

The latter question was answered by Khoussainov and Montalbán [1] in the affirmative. They generalized the construction of the random graph to allow the theory to code true arithmetic. We will answer the former question also in the affirmative. To answer the question, we construct an $\aleph_{1}$-categorical, in fact strongly minimal, theory in a finite language with no level of quantifier elimination even after any collection of parameters is named. This cannot be achieved with a disintegrated theory [6]. In fact, any disintegrated theory with a recursive model is Turing below $0^{\prime \prime}$. This bound also holds for modular strongly minimal expansions of groups 2 .

Received by the editors August 8, 2011 and, in revised form, October 11, 2011.

2010 Mathematics Subject Classification. Primary 03C98, 03D99. 
and trivially for field-like expansions of algebraically closed fields, which are all definitional expansions 14 . Thus the result cannot be achieved with the canonical examples of strongly minimal theories satisfying the Zilber trichotomy. Due to this, we construct the theory via a Hrushovski construction.

In fact, we will show the stronger result that if $\mathbf{d}$ is any tt-degree reducible to $0^{(\omega)}$, then there exists a strongly minimal theory $T \in \mathbf{d}$ in a finite language all of whose models are (uniformly) recursively presentable.

There will be two components to the proof of this recursive model-theoretic result. One, the infinite language version of the Hrushovski amalgamation construction [1, as presented in section 1.1 is a model-theoretic tool for building strongly minimal theories. The other, the Ash-Knight metatheorem [3] as presented in section 1.2 , is the recursion-theoretic tool to manage $0^{(\omega)}$-level information. First we will define the theory by the model-theoretic construction, and then we will verify by use of the metatheorem that all of the countable models of the theory have recursive presentations.

In section 4, we will similarly show that if $\mathbf{d}$ is any tt-degree below $0^{(\omega)}$, then there exists an $\aleph_{0}$-categorical theory $T \in \mathbf{d}$ in a finite language whose countable model is recursively presentable. The proof follows very similar lines, where we replace the Hrushovski amalgamation by a Fraïssé amalgamation and we replace the metatheorem argument with a theorem of Knight.

1.1. Infinite language Hrushovski constructions. This section is a summary of sections 2 and 3 of [1, which in turn is an adaptation of Hrushovski's original construction 9] to an infinite language. There is no new content in this section, and it is included for self-containment of this paper. The key concept of a Hrushovski construction is to fix a function $\delta$ on finite $L$-structures which will serve as an approximation to their dimension. The true dimension of any finite set $A$ will be $d(A)=\min \{\delta(B) \mid B \supseteq A\}$. We will then form a model by amalgamating finite $L$-structures as much as possible while ensuring that no finite set has negative dimension and that any extension of dimension 0 has at most a fixed finite number of copies.

Let $L$ be a countable relational language. For ease of notation, we assume $L$ contains only ternary relation symbols, say with signature $\left\{R_{i} \mid i \in I\right\}$. In the structure we generate, we ensure that each relation symbol is symmetric and holds only on distinct tuples. For a finite $L$-structure $A$, we write $|R(A)|$ for the number of tuples from $A$ on which $R$ holds. The following definitions are standard to Hrushovski constructions, and we will use them throughout.

Definition 1. For any finite $L$-structures $A$ and $B$ and infinite $L$-structure $D$, we define:

- $\delta(A)=|A|-\sum_{i \in I}\left|R_{i}(A)\right|$.

- $\delta(B / A)=\delta(A \cup B)-\delta(A)$.

- If $A \subseteq B$, we set $\delta(A, B)=\min \{\delta(C) \mid A \subseteq C \subseteq B\}$.

- Similarly, if $A \subseteq D$, we set $\delta(A, D)=\min \{\delta(C) \mid A \subseteq C \subset D, C$ finite $\}$.

- If $A \subseteq B$, we say that $A$ is strong in $B$ or $A \leq B$ if $\delta(A)=\delta(A, B)$.

We say that $A$ is strong in $D$ if $A \subseteq D$ and $A$ is strong in $C$ for each finite $A \subseteq C \subset D$.

- We say that $B$ is simply algebraic over $A$ if $A \cap B=\emptyset, A \leq A \cup B$, $\delta(B / A)=0$, and there is no proper subset $B^{\prime}$ of $B$ such that $\delta\left(B^{\prime} / A\right)=0$. 
- We say that $B$ is minimally simply algebraic over $A$ if $B$ is simply algebraic over $A$ and there is no proper subset $A^{\prime}$ of $A$ such that $B$ is simply algebraic over $A^{\prime}$.

- We say that $A$ and $B$ are freely joined if $\delta(A \cup B)=\delta(A)+\delta(B)-\delta(A \cap B)$.

Note that $A$ and $B$ are freely joined if and only if all relations on $A \cup B$ are on tuples entirely from $A$ or entirely from $B$. The following are key lemmas which verify that $\delta$ and $\leq$ act as expected.

Lemma 2 (Submodularity Property). For any finite L-structures $A, B \subseteq C, \delta(A \cup$ $B) \leq \delta(A)+\delta(B)-\delta(A \cap B)$.

Lemma 3. Let $A$ be a finite L-substructure of $N$. Suppose $A \leq N$.

(1) $\delta(X \cap A) \leq \delta(X)$ whenever $X \subseteq N$ is finite.

(2) $\delta\left(A^{\prime}, A\right)=\delta\left(A^{\prime}, N\right)$ whenever $A^{\prime} \subseteq A$.

(3) In particular, if $A^{\prime} \leq A \leq N$, then $A^{\prime} \leq N$.

Lemma 4. If $X, A$, and $B$ are finite $L$-structures such that $A \subseteq B$, then $\delta(X / A \cup$ $(X \cap B)) \geq \delta(X / B)$. In particular, if $X \cap B=\emptyset$, then $\delta(X / A) \geq \delta(X / B)$.

Up to this point, the definitions and lemmas have all been as in 9 . The following definition is necessary to allow for the infinite language. We need the condition that we bound the number of extensions of relative dimension 0 to be first order. Since the language is infinite, it would not be first order to bound the number of extensions $Y \supset X$ isomorphic to a particular pair $B \supset A$. Instead, we bound the number of extensions $Y$ over $X$ enough like $B \supset A$ that the relations showing $\delta(B / A)=0$ suffice to witness that $\delta(Y / X) \leq 0$. This idea is the content of the next definition.

Definition 5. - For any disjoint $L$-structures $A$ and $B$, we write $t p_{\text {r.q.f. }}(B / A)$ for the set $\left\{R_{i}(\bar{x}) \mid \bar{x} \subseteq(B \cup A)^{3} \backslash A^{3}, i \in I\right.$, and $R_{i}(\bar{x})$ holds $\}$. We call this set the relative quantifier-free type of $B$ over $A$.

- Let $L_{B / A}$ be the language generated by $\left\{R_{i} \mid \exists \bar{x} \in(B \cup A)^{3} \backslash A^{3}\left(R_{i}(\bar{x})\right)\right\}$, i.e., the language appearing in $t p_{\text {r.q.f. }}(B / A)$.

- Suppose $Y$ and $X$ are finite $L$-structures such that $Y$ is minimally simply algebraic over $X$ and that $B$ and $A$ are finite $L$-structures such that $\left.t p_{\text {r.q.f. }}(B / A)\right|_{L_{Y / X}}=t p_{\text {r.q.f. }}(Y / X)$ and $t p_{\text {q.f. }}(A)=t p_{q . f .}(X)$. In other words, $A$ is isomorphic to $X$ and up to the language $L_{Y / X}, B / A$ is isomorphic to $Y / X$. Then we say that the extension $B$ over $A$ is of the form of $Y$ over $X$.

We will define the amalgamation class with the two conditions that all finite sets have non-negative dimension and that a uniform bound holds on the number of extensions over a set $A$ of the form of $Y$ over $X$. To do so, we fix a bound function $\mu$ with the following properties:

- $\mu$ is a function from pairs of $L$-structures to $\omega$ such that $\mu(Y, X)$ depends only on the atomic type of the pair $(Y, X)$.

- For any $Y$ and $X, \mu(Y, X) \geq|X|$.

- If $\Gamma$ is a relative quantifier-free type, there exists a sublanguage $L^{\prime}$ of $L$ with finite subsignature such that whenever $t p_{\text {r.q.f. }}(Y / X)=\Gamma=t p_{\text {r.q.f. }}\left(Y^{\prime} / X^{\prime}\right)$ and $\left.t p_{q . f .}(X)\right|_{L^{\prime}}=\left.t p_{\text {q.f. }}\left(X^{\prime}\right)\right|_{L^{\prime}}$, then $\mu(Y, X)=\mu\left(Y^{\prime}, X^{\prime}\right)$.

The first condition is necessary for $\mu$ to be well defined, the second condition is necessary for the combinatorics of Lemma 8, and the last condition is again necessary to ensure that $\mu$ is a first-order bound. 
Definition 6. Let $\mathcal{C}=\mathcal{C}_{\mu}$ be the class of finite $L$-structures $C$ such that the following hold:

- If $A \subseteq C$, then $\delta(A) \geq 0$.

- Let $Y / X$ be a minimally simply algebraic extension. Let $B_{1}, \ldots, B_{n}, A$ be disjoint subsets of $C$ such that $B_{i} / A$ is of the form of $Y / X$ for each $i$. Then $n \leq \mu(Y, X)$.

To generate a model from $\mathcal{C}$, we verify that $\mathcal{C}$ has a strong amalgamation property. To see this, some combinatorics must be done. The key to the combinatorics is the following lemma, which will be used again in section 3 .

Lemma 7. Let $A, B_{1}, B_{2}$ be L-structures such that any substructure has nonnegative $\delta$-value, $A=B_{1} \cap B_{2}$, and $A \leq B_{1}$. Let $E$ be the free-join of $B_{1}$ with $B_{2}$ over $A$. Suppose $C^{1}, \ldots, C^{r}, F$ are disjoint substructures of $E$ such that each $C^{i}$ is minimally simply algebraic over $F$ and the structures $C^{i}$ and $C^{j}$ are isomorphic over $F$ for each $1 \leq i, j \leq r$. Then one of the following holds:

(1) One of the $C^{i}$ is contained in $B_{1} \backslash A$ and $F \subseteq A$.

(2) Either $F \cup \bigcup_{i=1}^{r} C^{i} \subseteq B_{2}$ or both $F \cup \bigcup_{i=1}^{r} C^{i} \subseteq B_{1}$ and one of the $C^{i}$ is contained in $B_{1} \backslash A$.

(3) $r \leq \delta(F)$.

(4) For one $C^{j}$, setting $X=(F \cap A) \cup\left(C^{j} \cap B_{2}\right), \delta(X / X \cap A)<0$. Further, one of the $C^{j}$ is contained in $B_{1} \backslash A$. (Note that this cannot happen if $A \leq B_{2}$ by Lemma 3.)

Proof. A careful reading of Lemma 3 of [9] will show that this is indeed what is proved there.

Lemma 8 (Strong Amalgamation Lemma). Suppose $A, B, C \in \mathcal{C}, A \leq B, A \leq C$. Then there exists $D \in \mathcal{C}$ such that $C \leq D$, and an embedding $g: B \rightarrow D$ such that $g(B) \leq D$ and $g(A)=\left.i d\right|_{A}$.

Proof. We refer the reader to Lemma 13 of [1].

The following theorem summarizes the results of section 3 of 1 .

Theorem 9. Amalgamation of $\mathcal{C}$ yields a unique countable generic amalgam $\mathcal{M}$. This $\mathcal{M}$ is saturated and strongly minimal. For $a \in \mathcal{M}$ and finite $B \subset \mathcal{M}, a \in$ $\operatorname{acl}(B)$ if and only if $d(a B)=d(B)$. Thus the algebraic dimension of a finite $B \subset M$ is $d(B)$. (Recall that $d(X)=\min \{\delta(Y) \mid Y \supseteq X, Y$ finite $\}$.)

1.2. The Ash-Knight metatheorem. This section entirely follows Ash-Knight [3] (see pg. 236), with the notational exception that the set which we call $V$ is there referred to as $L$. To maintain as much of the Ash-Knight notation as possible, we still refer to elements of $V$ as $l$ 's.

Let $V$ and $U$ be recursively enumerable sets, $E$ be a partial recursive enumeration function on $V$, and let $P$ be a recursively enumerable alternating tree on $V$ and $U$ made up of non-empty finite sequences which all start with the same $\hat{l} \in V$. Let $\left(\leq_{n}\right)_{n \in \omega}$ be uniformly recursively enumerable binary relations on $V$.

We define the tuple $\left(V, U, \hat{l}, P, E,\left(\leq_{n}\right)_{n<\omega}\right)$ to be an $\omega$-system if it satisfies the following properties:

(1) $\leq_{n}$ is reflexive and transitive for all $n<\omega$.

(2) $l \leq_{n} l^{\prime} \Rightarrow l \leq_{m} l^{\prime}$ for $m<n<\omega$. 
(3) If $l \leq_{0} l^{\prime}$, then $E(l) \subseteq E\left(l^{\prime}\right)$.

(4) If $\tau^{\wedge} u \in P$, where $\tau$ has length $2 n+1$ ending in $l^{0} \in V$ and

$$
l^{0} \leq_{n_{0}} l^{1} \leq_{n_{1}} \ldots \leq_{n_{t-1}} l^{t}
$$

for $n>n_{0}>\ldots>n_{t}$, then there exists $l^{*}$ such that $\tau u l^{*} \in P$ and $l^{i} \leq_{n_{i}} l^{*}$ for each $0 \leq i \leq t$.

Theorem 10 (Ash-Knight Metatheorem, 3, Thm. 14.4] with $\alpha=\omega)$. Let $(V, U, \hat{l}$, $\left.P, E,\left(\leq_{n}\right)_{n<\omega}\right)$ be an $\omega$-system, and let $q$ be a uniformly $0^{(n)}$ instruction function for $P$ (i.e., uniformly in $n, q$ computes $u_{n} \in U$ from input $\hat{l} u_{0} l_{0} u_{1} l_{1} \ldots l_{n-1}$ using oracle $\left.0^{n}\right)$. Then there is a path $\pi=\hat{l} u_{0} l_{0} u_{1} l_{1} \ldots$ through $P$ which agrees with the instruction function $q$ such that $E(\pi)$ is recursively enumerable.

We will use the metatheorem to construct structures whose atomic diagrams are recursively enumerable, i.e., recursive structures. Note that the proof that a particular $\omega$-system satisfies property (4) contains all the details as to how injury is handled in the construction.

\section{OUR THEORY}

Using the construction outlined in section 1.1, we need only specify a language $L$ and a function $\mu$ to produce a strongly minimal theory $\operatorname{Th}(\mathcal{M})$. The theory we seek will be a reduct of this one. We specify $L=\left\{R_{i} \mid i \in \omega\right\}$ with each symbol ternary.

The function $\mu$ will be our tool to encode recursion-theoretic information into our theory. Given any tt-degree $\mathbf{d} \leq_{\mathbf{t t}} \mathbf{0}^{(\omega)}$, fix a set $S \in \mathbf{d}$ with the property that whether $i$ is in $S$ is uniformly answered by $0^{(i)}$. Every tt-degree below $0^{(\omega)}$ contains such a set. We want to build a theory $T$ so that $T \equiv_{\mathrm{tt}} S$, so we will encode whether $i \in S$ into the bound for some minimally simply algebraic extension.

We fix a particular minimally simply algebraic extension $H$ over a three-element set $G$ such that $t p_{\text {r.q.f. }}(H / G)$ involves a single ternary relation symbol. We fix $k=|G \cup H|$. For $i \geq 1$, we write $\Gamma_{i}$ for the relative quantifier-free type formed by replacing the relation symbol in $t p_{\text {r.q.f. }}(H / G)$ by $R_{i-1}$. If $B$ and $A$ are finite $L$-structures such that $\left.t p_{\text {r.q.f. }}(B / A)\right|_{R_{i-1}}=\Gamma_{i}$, then we say that the extension $B$ over $A$ is a $\Gamma_{i}$-extension. We want to code whether or not $i \in S$ into the number of allowed $\Gamma_{i}$-extensions. This is the motivation for the difference between lines 3 and 4 of the following definition. The difference of line 2 with that of 3 or 4 and the appearance of $2^{k}$ throughout is to ensure that each $R_{i}$ is definable in terms of $R_{i-1}$ as in Lemma 13 .

Definition 11. Let $B$ be minimally simply algebraic over $A$. Then

$$
\mu(B, A)= \begin{cases}|A|+2^{k} & \text { if } B \text { over } A \text { is not a } \Gamma_{i} \text {-extension for any } i, \\ |A|+2^{k}+2 & \text { if } B \text { over } A \text { is a } \Gamma_{i} \text {-extension and } \neg R_{i}(A), \\ |A|+2^{k}+1 & \text { if } B \text { over } A \text { is a } \Gamma_{i} \text {-extension, } R_{i}(A), \text { and } i \in S, \\ |A|+2^{k} & \text { if } B \text { over } A \text { is a } \Gamma_{i} \text {-extension, } R_{i}(A), \text { and } i \notin S .\end{cases}
$$

We fix $T^{+}=\operatorname{Th}(\mathcal{M})$ to be the strongly minimal $L$-theory of the amalgam of $\mathcal{C}_{\mu}$ as in section 1.1. Our immediate task is to show that in $T^{+}$each $R_{i}$ is definable in terms of $R_{i-1}$ and that $T^{+}$encodes $S$. To do this, we need the following form of the algebraic amalgamation lemma specifically for $\Gamma_{i}$. It is for the combinatorics in the proof of this lemma that the $2^{k}$ term appears in $\mu$. 
Lemma 12 (Algebraic Amalgamation Lemma for $\Gamma_{i}$ ). Suppose $A, B_{1}, B_{2} \in \mathcal{C}$, $A=B_{1} \cap B_{2}$, and $B_{1} \backslash A$ is simply algebraic over $A$. Suppose further that $B_{1} \backslash A$ is minimally simply algebraic over $A^{\prime} \subseteq A$, and $t p_{\text {r.q.f. }}\left(B_{1} \backslash A / A^{\prime}\right)=\Gamma_{i}$. Then the free-join of $B_{1}$ with $B_{2}$ over $A$ is a member of $\mathcal{C}$ unless $B_{2}$ contains $\mu\left(B_{1} \backslash A, A^{\prime}\right)$ disjoint $\Gamma_{i}$-extensions over $A^{\prime}$.

Proof. Suppose the free-join $E$ is not in $\mathcal{C}$. This means that there are $C^{1}, \ldots, C^{n}$, $F$ contained in $E$ and a pair $(Y, X)$ such that each $C^{j}$ over $F$ is of the form of $Y$ over $X$ and $n>\mu(Y, X)$. Restricting $E$ to the language $L_{Y / X}$, we see that each of the $C^{j}$ are minimally simply algebraic over $F$ in the same way. Here we have the same setup as in Hrushovski's algebraic amalgamation lemma ([9], Lemma 3). Claims 1-3 and case 1 of Hrushovski's algebraic amalgamation lemma hold exactly as proved there. Case 1 leads to the exception in this lemma. In case 2, we need only count the number of $C^{j}$ which are entirely contained in $B_{1} \backslash A$. There are certainly fewer than $2^{\left|\left(B_{1} \backslash A\right) \cup A^{\prime}\right|}=2^{k}$ such $C^{j}$. Thus, $n \leq|F|+2^{k} \leq \mu(Y, X)$.

We will write $\Gamma_{i}(\bar{y}, \bar{x})$ to denote the first-order formula designating that $\bar{y}$ over $\bar{x}$ is a $\Gamma_{i}$-extension. Note that the formula $\Gamma_{i}(\bar{y}, \bar{x})$ involves only the relation symbol $R_{i-1}$.

Lemma 13. For every $i \geq 1, \mathcal{M} \models R_{i}(\bar{x}) \leftrightarrow \neg \exists^{5+2^{k}} \bar{y}\left(\Gamma_{i}(\bar{y}, \bar{x})\right.$ ). (We write $\exists^{m} \bar{y} \phi(\bar{y})$ to represent the formula stating that there exist $m$ disjoint tuples $\bar{y}$ satisfying $\phi$.)

Proof. The rightward direction follows from the fact that any finite substructure of $\mathcal{M}$ is an element of $\mathcal{C}$. If the rightward direction did not hold, then we would be violating the $\mu$-bound for $\Gamma_{i}$-extensions.

The leftward direction follows from the previous lemma. Suppose $\neg R_{i}(\bar{x})$ holds. Let $B$ be such that $\bar{x} \subseteq B \leq \mathcal{M}$. Repeated application of the previous lemma amalgamating a $\Gamma_{i}$-extension with $B$ over $\bar{x}$ shows that there is a $C \in \mathcal{C}$ such that $B \leq C$ and $C$ contains $5+2^{k}$ disjoint $\Gamma_{i}$-extensions over $\bar{x}$. The fullness of the amalgamation of $\mathcal{M}$ (i.e., whenever $B \leq M$ and $B \leq C$, there is an embedding $f: C \rightarrow M$ so that $f(C) \leq M$ and $f \mid B=i d_{B}$; this is property 3 in [1]) guarantees that this $C$ embeds in $\mathcal{M}$ over $B$.

Since the first-order formula $\Gamma_{i}(\bar{y}, \bar{x})$ is defined using only the relation $R_{i-1}$, we see that all of the $R_{i}$ are definable via the relation $R_{0}$.

Definition 14. Let $T=\left.T^{+}\right|_{R_{0}}$.

By Lemma 13, $T^{+}$is a definitional expansion of $T$. This $T$ is going to be the theory referenced in our main theorem. Note that the defining formulae for $R_{i}$ from $R_{0}$ do not depend on $S$ and are uniformly recursive, so $T \equiv_{\mathrm{tt}} T^{+}$. The following lemma shows that $T^{+}$, and thus $T$, tt-computes $S$.

Lemma 15. For every $i \geq 1, i \in S$ if and only if $\mathcal{M} \models \forall \bar{x} \exists^{4+2^{k}} \bar{y}\left(\Gamma_{i}(\bar{y}, \bar{x})\right)$.

Proof. There are as many disjoint $\Gamma_{i}$-extensions in $\mathcal{M}$ over $\bar{x}$ as $\mu$ allows, by the same argument as in Lemma 13. If $i \in S$, then $\mu$ always allows at least $4+2^{k}$ extensions. If $i \notin S$ and $R_{i}(\bar{x})$, then $\mu$ allows only $3+2^{k} \Gamma_{i}$-extensions over $\bar{x}$, witnessing that $\mathcal{M} \models \neg \forall \bar{x} \exists^{4+2^{k}} \bar{y}\left(\Gamma_{i}(\bar{y}, \bar{x})\right)$. 
This shows that $S \leq_{\mathrm{tt}} T^{+} \equiv_{\mathrm{tt}} T . T$ is also recursive in $S$ (properties 2 and $3^{\prime \prime}$ from [1] are explicit axiom schemata for $T$ which are recursive in $\mathcal{C}$, which in turn is recursive in $S$ ), and since the construction works for any $S$, this computation is total, yielding $S \equiv_{\mathrm{tt}} T$. All that remains is to show that each countable model of $T$ is recursive.

\section{Constructing the Countable models of $T$}

Before using the metatheorem to recursively construct models of $T$, we recall the construction of a model of $T$ ignoring the recursion-theoretic issues. The following will construct a $k$-dimensional model of $T^{+}$, i.e., a $k$-dimensional model of $T$.

Stage 0: Let $M_{0}$ be the structure with $k$ elements and no relations holding between them.

Stage $s>0$ : We are given $M_{s-1}$, the structure constructed at the previous stage. List the first $s$ minimally simply algebraic extensions over subsets of $M_{s-1}$ : $B_{1} / A_{1}, \ldots, B_{s} / A_{s}$. If the free amalgam of $B_{1}$ with $M_{s-1}$ over $A_{1}$ is in $\mathcal{C}$, replace $M_{s-1}$ with this amalgam. Repeating this procedure for $j \leq s$ gives a new structure, which we call $M_{s}$.

This gives a chain of structures $M_{0} \leq M_{1} \leq \ldots \leq M_{k} \leq \ldots \leq \bigcup_{i} M_{i}$, where $\bigcup_{i} M_{i}$ is the $k$-dimensional model of $T^{+}$. We wish to employ this construction, but we cannot recursively compute $\mu$, so we cannot recursively tell whether an amalgam is in $\mathcal{C}$. In fact, the $k$-dimensional model of $T^{+}$will not be recursive. We will rather work with approximations to $S$, thus to $\mu$ and $\mathcal{C}$, and will build approximations to a model of $T^{+}$, but we will injure our $R_{i}$-assignments when our approximation to the question of whether $i \in S$ changes. Of course, we will use the metatheorem to do this coherently for all $i$. Recall that whether $i \in S$ is uniformly answered by $0^{(i)}$.

We use the Ash-Knight Metatheorem to construct the $k$-dimensional model of $T$ by defining an $\omega$-system $\left(V, U, \hat{l}, P, E,\left(\leq_{n}\right)_{n \in \omega}\right)$. Throughout the construction, we will be working with various estimates to the set $S$. These estimates will be represented by elements of $2^{<\omega}$. Given the estimate $\sigma$, we define $\mu_{\sigma}$ and $\mathcal{C}_{\sigma}$, the corresponding approximations to $\mu$ and $\mathcal{C}$.

Definition 16. Let $\sigma$ be an element of $2^{<\omega}$ where length $(\sigma)=n+1(\sigma(0)$ is never referenced, so this index is off by one).

We define $L_{\sigma}=L_{n}$ to be the language generated by the relation symbols $\left\{R_{i} \mid i<\right.$ $n\}$.

For $B$ a minimally simply algebraic extension over $A$, let

$$
\mu_{\sigma}(B, A)= \begin{cases}|A|+2^{k} & \text { if } B \text { over } A \text { is not a } \Gamma_{i} \text {-extension for any } i \in \omega, \\ |A|+2^{k}+2 & \text { if } B \text { over } A \text { is a } \Gamma_{i} \text {-extension and } \neg R_{i}(A), \\ |A|+2^{k}+1 & \text { if } B \text { over } A \text { is a } \Gamma_{i} \text {-extension, } R_{i}(A), \text { and } \sigma(i)=1, \\ |A|+2^{k} & \text { if } B \text { over } A \text { is a } \Gamma_{i} \text {-extension, } R_{i}(A), \text { and } \sigma(i)=0 .\end{cases}
$$

Note that $\mu_{\sigma}(B, A)$ is defined for any finite $L_{\sigma}$ extension, so the following is well defined. Let $\mathcal{C}_{\sigma}$ be the class of finite $L_{\sigma}$ structures $C$ such that the following conditions hold:

$$
\text { - } \delta(A) \geq 0 \text { for all } A \subseteq C \text {. }
$$


- Let $Y$ over $X$ be a minimally simply algebraic extension. Suppose $B_{1}, \ldots$, $B_{n}, A$ are disjoint subsets of $C$ such that each $B_{i}$ over $A$ is an extension of the form of $Y$ over $X$. Then $n \leq \mu_{\sigma}(Y, X)$.

Definition 17. Fix $k \in \omega$. We define $S_{k}$ to be the following system (which will construct the $k$-dimensional model of $T$ ):

- $V$ is the set of pairs $(M, \sigma)$, where $M$ is a finite $L$-structure whose universe is an initial segment of $\omega, \sigma \in 2^{<\omega}$, and $M \in \mathcal{C}_{\sigma}$. For $l \in V$, we write $l=\left(M_{l}, \sigma_{l}\right)$.

- $U$ is $2^{<\omega}$.

- $\hat{l}$ is the pair $(M, \sigma)$, where $M$ is the structure with $k$ elements and no relations and $\sigma$ is the trivial string of length 0 .

- $E(l)$ is the set of primitive (atomic and negations of atomic) statements true about $M_{l}$ in the language generated by the single relation symbol $R_{0}$.

- $l \leq_{n} l^{\prime}$ if the following conditions hold:

(1) $\left.\sigma_{l}\right|_{n}=\left.\sigma_{l^{\prime}}\right|_{n}$ (i.e., $\sigma_{l}(i)=\sigma_{l^{\prime}}(i)$ for $\left.i \leq n\right)$.

(2) The universe of $M_{l}$ is a subset of the universe of $M_{l^{\prime}}$.

(3) $\left.M_{l}\right|_{L_{n}} \leq\left. M_{l^{\prime}}\right|_{L_{n}}$. By this we mean that as $L_{n}$-structures $\left.M_{l}\right|_{L_{n}} \subseteq$ $\left.M_{l^{\prime}}\right|_{L_{n}}$ and it is a strong substructure.

- $P$ is the tree where $\hat{l} u_{0} l_{0} \ldots u_{t} l_{t} \in P$ if:

(1) For each $i, \sigma_{l_{i}}=u_{i}$.

(2) For each $i, M_{l_{i}} \leq M_{l_{i+1}}$.

(3) For each $i, \delta\left(M_{l_{i}}\right)=k$.

(4) Take a universal list of all minimally simply algebraic extensions in $L$ along with sets the extension could be over; call it List. For the first $i$ entries on List, the free-join of the extension with $M_{l_{i}}$ is not in $\mathcal{C}_{\sigma_{l_{i}}}$. (The last item says that all the copies of each of the first $i$ extensions which are allowed in $\mathcal{C}_{\sigma_{l_{i}}}$ occur already in $M_{l^{i}}$.)

Theorem 18. $S_{k}$ is an $\omega$-system.

Proof. We focus on the difficult condition.

Suppose $\tau u \in P$, length $(\tau)=2 n+1, \tau$ ends in $l^{0}$, and

$$
l^{0} \leq_{n_{0}} l^{1} \leq_{n_{1}} \ldots \leq_{n_{t-1}} l^{t}
$$

for $n>n_{0}>n_{1}>\ldots>n_{t-1}>n_{t}$. Without loss of generality, we assume that $n_{0}=n-1$. We need to show that there exists an $l^{*}$ such that $\tau u l^{*} \in P$, and for each $i, l^{i} \leq_{n_{i}} l^{*}$. First we will define an auxiliary structure $l^{\#}=(\mathcal{N}, \sigma)$ which will handle the injury occurring in this sequence of $l$ 's. To form $\mathcal{N}$, we keep the occurrences of relation symbols from the $M_{l^{j}}$ to which we are committed and forget all of the others. Some combinatorics will be required to verify that $N \in \mathcal{C}_{u}$. We will then extend $l^{\#}$ to an $l^{*}$ which has the right dimension and contains amalgamations of the required minimally simply algebraic extensions from $\mathcal{C}_{u}$. To avoid notation such as $M_{l^{j}}$, we write $l^{j}=\left(M_{j}, \sigma_{j}\right)$.

Let $l^{\#}$ be the pair $(\mathcal{N}, \sigma)$, defined as follows: $\sigma=u$ and $\mathcal{N}$ has the same universe as $M_{t}$. Let $\bar{x}$ be a tuple in $\mathcal{N}$. We now describe whether or not $R_{i}$ holds on $\bar{x}$. Let $m$ be least such that $\bar{x} \subseteq M_{m}$. Then $R_{i}$ holds on $\bar{x}$ in $\mathcal{N}$ if and only if $R_{i}$ is in $L_{n_{m}}$ and holds on $\bar{x}$ in $M_{m}$.

We will write $\mathcal{N}_{i}$ for the substructure of $\mathcal{N}$ with the same universe as $M_{i}$, and we will write $\mathcal{L}_{i}$ for $L_{n_{i}}$. 
Claim 19. $\left.M_{i}\right|_{\mathcal{L}_{i}}=\left.\mathcal{N}_{i}\right|_{\mathcal{L}_{i}}$; i.e., for each relation in $\mathcal{L}_{i}$ and every tuple in $M_{i}$, the relation holds in $M_{i}$ if and only if it holds in $\mathcal{N}$.

Proof. For each $j \leq i, l^{j} \leq_{n_{i-1}} l^{i}$. In particular, $l^{j} \leq_{n_{i}} l^{i}$. Let $\bar{x}$ be any tuple in $M_{i}$ and let $m$ be minimal such that $\bar{x} \subseteq M_{m}$. Then $m \leq i$, so $l^{m} \leq_{n_{i}} l^{i}$. Thus for $R \in \mathcal{L}_{i}, R(\bar{x})$ holds in $M_{m}$ if and only if it holds in $\mathcal{N}$ (by definition of $\mathcal{N}$ ), and the first condition is equivalent to $R(\bar{x})$ holding in $M_{i}$, since $l^{m} \leq_{n_{i}} l^{i}$.

In particular, $\mathcal{N}_{0}=M_{0}$ since $n_{0}=n-1$.

Lemma 20. $l^{\#} \in V$.

Proof. We need to verify that $\mathcal{N} \in \mathcal{C}_{\sigma}$. We do this by verifying each condition in the definition of $\mathcal{C}_{\sigma}$.

(1) $\delta(A) \geq 0$ for all $A \subseteq \mathcal{N}$.

Proof. Let $A$ be a subset of $\mathcal{N}$. Let $A_{i}=A \cap \mathcal{N}_{i}$. We need to show that $\delta\left(A_{t}\right) \geq 0$. We achieve this by showing that $\delta\left(A_{i+1} / A_{i}\right) \geq 0$ for each $i$. This suffices since $\delta\left(A_{t}\right)=\delta\left(A_{t} / A_{t-1}\right)+\delta\left(A_{t-1} / A_{t-2}\right)+\ldots+\delta\left(A_{1} / A_{0}\right)+\delta\left(A_{0}\right)$ and $A_{0}$ is a subset of $\mathcal{N}_{0}=M_{0}$, hence has non-negative $\delta$-value.

$\delta\left(A_{i+1} / A_{i}\right)$ is $\left|A_{i+1} \backslash A_{i}\right|$ - (the number of relations holding in $A_{i+1}$ involving at least one element in $\left.A_{i+1} \backslash A_{i}\right)$. Consider $B$ to be the subset of $M_{i+1}$ with the same underlying set as $A_{i+1}$. Since $\left.M_{i}\right|_{\mathcal{L}_{i}} \leq\left. M_{i+1}\right|_{\mathcal{L}_{i}}$, $\delta\left(\left.B\right|_{\mathcal{L}_{i}} /\left.\left(B \cap M_{i}\right)\right|_{\mathcal{L}_{i}}\right) \geq 0$, but $\delta\left(\left.B\right|_{\mathcal{L}_{i}} /\left.\left(B \cap M_{i}\right)\right|_{L_{i}}\right) \leq \delta\left(A_{i+1} / A_{i}\right)$, as every relation counting on the right counts on the left as well. Thus, each summand is non-negative and $\delta(A) \geq 0$.

(2) If $C^{1}, \ldots, C^{n}, F$ are disjoint subsets of $\mathcal{N}$, and each $C^{j}$ over $F$ is of the form of $Y$ over $X$, a minimally simply algebraic extension, then $n \leq \mu_{\sigma}(Y, X)$.

Proof. We proceed by induction to show that the condition holds for each $\mathcal{N}_{i}$. The condition holds on $\mathcal{N}_{0}$, as this is just $M_{0}$ and $\mu_{\sigma}$ agrees with $\mu_{\sigma_{0}}$. Supposing the condition holds for $\mathcal{N}_{s-1}$, we will show that the condition holds on $\mathcal{N}_{s}$ as well.

The proof follows via Lemma 7. Suppose $C^{1}, \ldots, C^{n}, F$ are disjoint subsets of $\mathcal{N}_{s}$, and each $C^{j}$ over $F$ is of the form of $Y$ over $X$. Since $\mathcal{N}_{s-1} \leq \mathcal{N}_{s}$, we apply Lemma 7 with $B_{1}=\left.\mathcal{N}_{s}\right|_{L_{Y / X}}, A=B_{2}=\left.\mathcal{N}_{s-1}\right|_{L_{Y / X}}$. There are 4 cases to consider. In one case, $r \leq|X|<\mu_{\sigma}(Y, X)$. In each of the other cases, one $C^{j}$ is entirely contained in $\mathcal{N}_{s} \backslash \mathcal{N}_{s-1}$.

In the case that one $C^{j}$ is entirely contained in $\mathcal{N}_{s} \backslash \mathcal{N}_{s-1}, t p_{\text {r.q.f. }}(Y / X)$ only includes relations from the language $\mathcal{L}_{s}$. There are a number of possibilities to consider:

Case 1: $Y$ over $X$ is not a $\Gamma_{i}$-extension for any $i$.

Let $F_{0}$ be the subset of $M_{s}$ with underlying set the same as $F$. Since $\left.M_{s}\right|_{\mathcal{L}_{s}}=\left.\mathcal{N}_{s}\right|_{\mathcal{L}_{s}}$, we see that each of the $C^{j}$ over $F$, looked at as subsets of $M_{s}$, is of the form of $Y$ over $X^{\prime}$, where $X^{\prime} \cong F_{0}$. Since for non- $\Gamma_{i}$-extensions, $\mu_{\sigma}(Y, X)$ only depends on $|X|$, and since $M_{s}$ satisfies the property for $\mu_{\sigma_{s}}$, we have $n \leq \mu_{\sigma_{s}}\left(Y, X^{\prime}\right)=\mu_{\sigma}(Y, X)$.

Case 2: $Y$ over $X$ is a $\Gamma_{i}$-extension and $\neg R_{i}(X)$.

In this case, we also look at the $C^{j}$ and $F$ as subsets of $M_{s}$. The $C^{j}$ are each $\Gamma_{i}$-extensions over $F$. If $R_{i}(F)$ is in $M_{s}$, then the number of $C^{j}$ is 
bounded by $\mu_{\sigma_{s}}\left(Y, X^{\prime}\right)$ (where $X^{\prime}$ is the same as $X$ but with $R_{i}(X)$ holding), which is even less than $\mu_{\sigma_{s}}(Y, X)$. If $\neg R_{i}(F)$ in $M_{s}$, then the number of $C^{j}$ is bounded by $\mu_{\sigma_{s}}(Y, X)$. Since $\left.\sigma_{s}\right|_{n_{s-1}}=\left.\sigma\right|_{n_{s-1}}, \mu_{\sigma_{s}}(Y, X)=\mu_{\sigma}(Y, X)$.

Case 3: $Y$ over $X$ is a $\Gamma_{i}$-extension and $R_{i}(X)$.

If $R_{i} \in \mathcal{L}_{s}$, then we have the corresponding fact in $M_{s}$, so we get the $\mu$-bound from the fact that $M_{s}$ satisfies the property for $\mu_{\sigma_{s}}$ and $\left.\sigma_{s}\right|_{n_{s-1}}=$ $\left.\sigma\right|_{n_{s-1}}$. Now suppose $R_{i} \notin \mathcal{L}_{s}$. Since $Y$ over $X$ is a $\Gamma_{i}$-extension, $R_{i-1} \in \mathcal{L}_{s}$. So $R_{i} \in \mathcal{L}_{s-1}$. Clearly, $R_{i}(F)$ implies that $F \subseteq \mathcal{N}_{s-1}$. But then $R_{i}(F)$ holds in $M_{s-1}$, and $l^{s-1} \leq_{n_{s-1}} l^{s}$, so $R_{i}(F)$ holds in $M_{s}$ as well. Again, we get the $\mu$-bound from $M_{s}$.

This concludes the inductive step, showing that $\mathcal{N}=\mathcal{N}_{t}$ satisfies the condition.

Claim 21. For each $i, l^{i} \leq_{n_{i}} l^{\#}$.

Proof. We verify the two properties. First we verify that $\left.\sigma_{i}\right|_{n_{i}}=\left.\sigma\right|_{n_{i}}$. We know that $l^{0} \leq_{n_{i-1}} l^{i}$, so $\left.\sigma\right|_{n_{i}}=\left.\sigma_{0}\right|_{n_{i}}=\left.\sigma_{i}\right|_{n_{i}}$.

Second we verify that $\left.M_{i}\right|_{\mathcal{L}_{i}} \leq\left.\mathcal{N}\right|_{\mathcal{L}_{i}}$. Claim 19 gives us that $\left.\left.M_{i}\right|_{\mathcal{L}_{i}} \subset \mathcal{N}\right|_{\mathcal{L}_{i}}$.

Now, let $X$ be a subset of $\left.\mathcal{N}\right|_{\mathcal{L}_{i}}$. We use the same argument as before (when we showed that $\delta(A) \geq 0$ for all $A \subseteq \mathcal{N})$. We need to show that $\delta\left(X /\left.\mathcal{N}_{i}\right|_{\mathcal{L}_{i}}\right) \geq 0$. For $i \leq j \leq t$, we write $X_{j}=\left.\left(\left(X \cap \mathcal{N}_{j}\right) \cup \mathcal{N}_{i}\right)\right|_{\mathcal{L}_{i}}$. Then we have $\delta\left(X /\left.\mathcal{N}_{i}\right|_{\mathcal{L}_{i}}\right)=$ $\delta\left(X_{t} / X_{t-1}\right)+\delta\left(X_{t-1} / X_{t-2}\right)+\ldots+\delta\left(X_{i+1} / X_{i}\right)$. As in the previous argument, each summand is non-negative, so $\delta\left(X /\left.\mathcal{N}_{i}\right|_{\mathcal{L}_{i}}\right) \geq 0$.

The only obstructions to $l^{\#}$ being what we need for $l^{*}$ is that it might not contain enough copies of the first $n$ minimally simply algebraic extensions, and perhaps $\delta(\mathcal{N})>k$. Extend $\mathcal{N}$ using only the thus far unused relation symbol $R_{n-1}$ to $\mathcal{N}^{\prime}$ so that $\delta\left(\mathcal{N}^{\prime}\right)=k, M_{0} \leq \mathcal{N}^{\prime}$, and $\mathcal{N}^{\prime} \in \mathcal{C}_{\sigma}$. Then proceed to extend $\mathcal{N}^{\prime}$ to $\mathcal{N}^{*}$ by amalgamating as many copies as allowed in $\mathcal{C}_{\sigma}$ of the first $n$ minimally simply algebraic extensions over $\mathcal{N}^{*}$. We set $l^{*}$ to be $\left(\mathcal{N}^{*}, u\right)$. By construction, $l^{i} \leq_{n_{i}} l^{\#} \leq n_{n_{i}} l^{*}$ and $\tau u l^{*} \in P$ as $l^{*} \in V, M_{0} \leq \mathcal{N}^{*}, \sigma_{l^{*}}=u$, and $\delta\left(\mathcal{N}^{*}\right)=k$. Having found this $l^{*}$, we have shown that $\left(V, U, \hat{l}, E, P,\left(\leq_{n}\right)_{n \in \omega}\right)$ is an $\omega$-system.

We have a uniformly $0^{(n)}$ instruction function for $u$, namely $u_{n}=\left.S\right|_{n}$, the string in $2^{<\omega}$ describing membership in $S$ for integers $\leq n$. Thus, the metatheorem gives us a run, i.e., a path $\pi=\hat{l} u_{0} l_{0} u_{1} l_{1} \ldots$ through $P$ consistent with this instruction function such that $E(\pi)$ is recursively enumerable. $E(\pi)$ gives us the $R_{0}$-atomic diagram of $\bigcup_{i} M_{l_{i}}$.

Theorem 22. The $k$-dimensional model of $T$ is recursively presentable.

Proof. It remains to show that the model $M=\bigcup_{i} M_{l_{i}}$ is isomorphic to the $k$ dimensional model of $T$.

By construction, $M$ satisfies properties 2 and $3^{\prime \prime}$ of [1]; thus $M \models T$. Furthermore, $\hat{l} \leq M$, so the dimension is at least $k$, and any finite $A \subseteq M$ is contained in some $M_{l_{i}}$, so has dimension $\leq \delta\left(M_{l_{i}}\right)=k$. Thus the dimension of $M$ is $k$.

Theorem 23. The saturated model of $T$ is recursively presentable. 
Proof. We use a similar $\omega$-system where instead of insisting that $\delta\left(M_{l_{i}}\right)=k$ in the definition of $P$, we insist that $\delta\left(M_{l_{i}}\right) \geq i$. To get $\mathcal{N}^{\prime}$ from $\mathcal{N}$ we add a new element not related to anything else. In the final model, we constructed it so that $1,2,3^{\prime}, 3^{\prime \prime}$ of [1] all hold, so the constructed model is the saturated model of $T$.

Putting together Lemma 15, Theorem 22] and Theorem 23, we conclude the main theorem.

Theorem 24. Let $\mathbf{d}$ be a tt-degree reducible to $0^{(\omega)}$. There exists a strongly minimal theory $T \in \mathbf{d}$ in a finite language such that each countable model of $T$ is recursively presentable.

Proof. We have chosen $S \in \mathbf{d}$ and constructed $T \equiv_{\mathrm{tt}} S$ so that all countable models of $T$ have recursive presentations.

Corollary 25. There exists a strongly minimal theory $T$ in a finite language so that $T \equiv_{T} 0^{(\omega)}$ and each countable model of $T$ is recursively presentable.

\section{THE $\aleph_{0}$-CATEGORICAL CASE}

As above, we fix a set $S$ such that whether $n \in S$ is uniformly recursive in $0^{(n)}$. We work at first in an infinite language to produce the theory; then we will take a reduct to a sublanguage with finite subsignature. Let $L$ be the language with signature $\{P, Q\} \cup\left\{R_{i} \mid i \geq 3\right\}$, where $P$ and $Q$ are binary relation symbols and each $R_{i}$ is $i$-ary. We will generate an $\aleph_{0}$-categorical theory via a Fraïssé construction (see [8], section 6.1) using the following amalgamation class $\mathcal{K}$.

Definition 26. Let $\mathcal{K}$ be the class of finite $L$-structures $C$ which satisfy the following properties:

- Each relation symbol is symmetric and holds only on tuples of distinct elements.

- If $i-10 \notin S$ or $i<10$, then $C$ satisfies

$$
\neg \exists \bar{x}, y, z\left[R_{i}(\bar{x}) \wedge P(y, z) \wedge \bigwedge_{\bar{w} \subset \bar{x},|\bar{w}|=i-2}\left(R_{i-1}(y, \bar{w}) \wedge R_{i-1}(z, \bar{w})\right)\right] .
$$

- If $i-10 \in S$, then $C$ satisfies

$$
\neg \exists \bar{x}, y, z\left[R_{i}(\bar{x}) \wedge Q(y, z) \wedge \bigwedge_{\bar{w} \subset \bar{x},|\bar{w}|=i-2}\left(R_{i-1}(y, \bar{w}) \wedge R_{i-1}(z, \bar{w})\right)\right] .
$$

To carry out a Fraïssé construction using $\mathcal{K}$, we must verify the following lemma:

Lemma 27. $\mathcal{K}$ satisfies $H P, J E P$, and $A P$.

Proof. Since $\mathcal{K}$ is defined via $\forall_{1}$ formulae, it satisfies HP.

Given two disjoint structures $B, C \in \mathcal{K}$, we see that the free-join of $B$ with $C$ over $\emptyset$ is in $\mathcal{K}$, so $\mathcal{K}$ satisfies JEP.

Lastly, given three structures $A, B, C \in \mathcal{K}$ where $A=B \cap C$, we will show that the free-join of $B$ with $C$ over $A$ is in $\mathcal{K}$, and thus $\mathcal{K}$ satisfies AP. Suppose the free-join is not in $\mathcal{K}$. Then there exists $i, \bar{x}, y, z$ witnessing this. We may assume $i-10 \in S$, so $\bar{x}, y, z$ satisfy:

$$
R_{i}(\bar{x}) \wedge Q(y, z) \wedge \bigwedge_{\bar{w} \subset \bar{x},|\bar{w}|=i-2}\left(R_{i-1}(y, \bar{w}) \wedge R_{i-1}(z, \bar{w})\right) .
$$


Since any two elements of $\bar{x} \cup\{y, z\}$ are related by some relation, the whole set is contained in either $B$ or $C$. Thus either $B \notin \mathcal{K}$ or $C \notin \mathcal{K}$, yielding a contradiction.

Now we use Fraïssé's theorem ([8], Theorem 6.1.2), which guarantees a countable ultra-homogeneous $L$-structure $\mathcal{M}$ with $\operatorname{Age}(\mathcal{M})=\mathcal{K}$. As $\mathcal{M}$ is ultra-homogeneous, it admits quantifier elimination. Thus, the number of $n$-types is bounded by the number of possible configurations of the finitely many relations in $L$ of arity $\leq n$, showing that $\operatorname{Th}(\mathcal{M})$ is $\aleph_{0}$-categorical. The following lemma allows the reduction to a finite sublanguage.

Lemma 28. Suppose $i>3$ :

If $i-10 \notin S$ or $i<10$, then

$$
\mathcal{M} \models R_{i}(\bar{x}) \leftrightarrow \neg \exists y, z\left[P(y, z) \wedge \bigwedge_{\bar{w} \subset \bar{x},|\bar{w}|=i-2}\left(R_{i-1}(y, \bar{w}) \wedge R_{i-1}(z, \bar{w})\right)\right] .
$$

Similarly, if $i-10 \in S$, then

$$
\mathcal{M} \models R_{i}(\bar{x}) \leftrightarrow \neg \exists y, z\left[Q(y, z) \wedge \bigwedge_{\bar{w} \subset \bar{x},|\bar{w}|=i-2}\left(R_{i-1}(y, \bar{w}) \wedge R_{i-1}(z, \bar{w})\right)\right] .
$$

Proof. The rightward direction follows via the fact that $\operatorname{Age}(M)=\mathcal{K}$. To show the leftward direction, take a tuple $\bar{x}$ such that $\mathcal{M} \models \neg R_{i}(\bar{x})$. By ultra-homogeneity of $\mathcal{M}$, it suffices to show that $\bar{x}$ embeds into an element of $\mathcal{K}$ where such a $y$ and $z$ exist. Consider the structure $A=\bar{x} \cup\{a, b\}$, where each of $a$ and $b$ are $R_{i-1}$-related to every $(i-2)$-element subset of $\bar{x}, a$ and $b$ are $P$-related $(Q$-related in the case of the second equivalence above), and no other relations hold involving $a$ or $b$. It is easy to verify that this structure is in $\mathcal{K}$.

Definition 29. Let $T=\left.\operatorname{Th}(\mathcal{M})\right|_{\left\{P, Q, R_{3}\right\}}$.

By the previous lemma, $\operatorname{Th}(\mathcal{M})$ is a definitional expansion of $T$. Further, from $T$ we can in a tt way determine which definition of $R_{i}$ is correct, and thus whether $i-10 \in S$. This shows that $T \geq_{\mathrm{tt}} S$. Further, by ultra-homogeneity of $T, T \leq_{\mathrm{tt}}$ $\mathcal{K} \leq_{\text {tt }} S$. It remains only to prove that the countable model of $T$ is recursively presentable.

Lemma 30. Uniformly in $n, T \cap \exists_{n}$ is computable in $0^{n-7}$.

Proof. We first show that any $\exists_{n}$ formula in $T$ is equivalent to a quantifier-free formula in the relations $\left\{P, Q, R_{i}\right\}_{i \leq 3+n}$. The proof proceeds by setting up the appropriate Ehrenfeucht-Fraïssé game (see [13, section 2.4) and seeing that ' $\exists$ loise' (the second player) has a winning strategy. The game is the standard EhrenfeuchtFraïssé game of length $n$ where we start with tuples $\bar{a}$ and $\bar{b}$ which have the same $\left\{P, Q, R_{i}\right\}_{i \leq 3+n}$-quantifier-free type. Then whichever tuple $\bar{c}$ ' $\forall$ belard' chooses, $\exists$ loise can choose a tuple $\bar{d}$ so that $\bar{a} \bar{c}$ and $\bar{b} \bar{d}$ satisfy the same $\left\{P, Q, R_{i}\right\}_{i \leq 3+n-1^{-}}$ quantifier-free type. Proceeding as such, $\exists$ loise wins the game of length $n$. 
This shows that any $\exists_{n}$ formula depends only on the relations $\left\{P, Q, R_{i}\right\}_{i \leq 3+n}$ ([13, Lemma 2.4.9). Thus, the $\exists_{n}$ formula $\exists \forall \ldots \phi(\bar{x})$ is equivalent to:

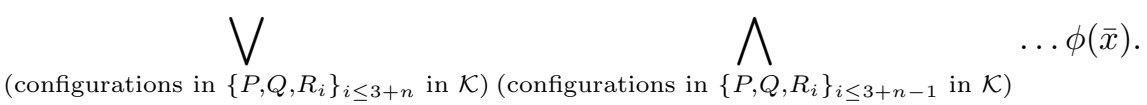

To verify whether this statement is true, we need only to be able to parse "in $\mathcal{K}$ " for configurations in the language $\left\{P, Q, R_{i}\right\}_{i<3+n}$. The conditions of being in $\mathcal{K}$ are then described recursively in $0^{3+n-10}$.

We use the following case of a theorem of Knight 12 to show that the countable model of $T$ is recursively presentable.

Theorem 31 (Knight). Let $T$ be an $\aleph_{0}$-categorical theory. If $T \cap \exists_{n+1}$ is $\Sigma_{n}^{0}$ uniformly in $n$, then $T$ has a recursive model.

Since $T$ is $\aleph_{0}$-categorical, Lemma 30 shows that $T$ satisfies the conditions of this theorem. Thus we conclude the promised theorem.

Theorem 32. Let $\mathbf{d}$ be a tt-degree reducible to $0^{(\omega)}$. Then there exists an $\aleph_{0}$ categorical theory $T \in \mathbf{d}$ in a finite language whose countable model is recursively presentable.

Proof. As above, $\mathbf{d}$ contains a set $S$ such that $n \in S$ is uniformly recursive in $0^{(n)}$. Applying the construction to this set $S$ yields an $\aleph_{0}$-categorical theory $T \in \mathbf{d}$ in a finite language whose countable model is recursively presentable.

Corollary 33. There exists an $\aleph_{0}$-categorical theory $T$ in a finite language whose countable model is recursively presentable and $T \equiv_{T} 0^{(\omega)}$.

\section{REFERENCES}

1. Uri Andrews, A new spectrum of recursive models using an amalgamation construction, J. Symbolic Logic 76 (2011), 883-896. MR2849250

2. Uri Andrews and Alice Medvedev, Recursive spectra of strongly minimal theories satisfying the Zilber trichotomy, To appear in Trans. Amer. Math. Soc.

3. C. J. Ash and J. F. Knight, Computable structures and the hyperarithmetical hierarchy, Studies in Logic and the Foundations of Mathematics, vol. 144, North-Holland Publishing Co., Amsterdam, 2000. MR 1767842 (2001k:03090)

4. Ekaterina Fokina, Arithmetic Turing degrees and categorical theories of computable models, Mathematical logic in Asia, World Sci. Publ., Hackensack, NJ, 2006, pp. 58-69. MR2294286 (2008f:03058)

5. Sergei S. Goncharov and Bakhadyr Khoussainov, Complexity of theories of computable categorical models, Algebra Logika 43 (2004), no. 6, 650-665, 758-759. MR 2135386 (2005m:03066)

6. Sergey S. Goncharov, Valentina S. Harizanov, Michael C. Laskowski, Steffen Lempp, and Charles F. D. McCoy, Trivial, strongly minimal theories are model complete after naming constants, Proc. Amer. Math. Soc. 131 (2003), no. 12, 3901-3912 (electronic). MR.1999939 (2004g:03054)

7. Leo Harrington, Recursively presentable prime models, J. Symbolic Logic 39 (1974), 305-309. MR.0351804(50:4292)

8. Wilfrid Hodges, A shorter model theory, Cambridge University Press, Cambridge, 1997. MR.1462612 (98i:03041)

9. Ehud Hrushovski, A new strongly minimal set, Ann. Pure Appl. Logic 62 (1993), no. 2, 147-166, Stability in model theory, III (Trento, 1991). MR1226304 (94d:03064)

10. Nazif G. Khisamiev, Strongly constructive models of a decidable theory, Izv. Akad. Nauk Kazah. SSR Ser. Fiz.-Mat. (1974), no. 1, 83-84, 94. MR0354344 (50:6824) 
11. Bakhadyr Khoussainov and Antonio Montalbán, A computable $\aleph_{0}$-categorical structure whose theory computes true arithmetic, J. Symbolic Logic 75 (2010), no. 2, 728-740. MR2648162 (2011g:03083)

12. Julia F. Knight, Nonarithmetical $\aleph_{0}$-categorical theories with recursive models, J. Symbolic Logic 59 (1994), no. 1, 106-112. MR1264967(95a:03045)

13. David Marker, Model theory. An introduction, Graduate Texts in Mathematics, vol. 217, Springer-Verlag, New York, 2002. MR 1924282(2003e:03060)

14. Bruno Poizat, MM. Borel, Tits, Zil'ber et le Général Nonsense, J. Symbolic Logic 53 (1988), no. 1, 124-131. MR.929379 (89c:03060)

Department of Mathematics, University of Wisconsin, 480 Lincoln Drive, Madison, WISCONSIN 53706

E-mail address: andrews@math.wisc.edu 